\title{
Realization of Universal Optimal Quantum Machines by Projective Operators and Stochastic Maps
}

\author{
F. Sciarrino, C. Sias, M. Ricci and F. De Martini \\ Istituto Nazionale per la Fisica della Materia, Dipartimento di Fisica, \\ Università di Roma "La Sapienza", Roma, 00185 - Italy
}

(November 30, 2018)

\begin{abstract}
Optimal quantum machines can be implemented by linear projective operations. In the present work a general qubit symmetrization theory is presented by investigating the close links to the qubit purification process and to the programmable teleportation of any generic optimal anti-unitary map. In addition, the contextual realization of the $N \rightarrow M$ cloning map and of the teleportation of the $N \rightarrow(M-N)$ universal NOT gate is analyzed by a novel and very general angular momentum theory. An extended set of experimental realizations by state symmetrization linear optical procedures is reported. These include the $1 \rightarrow 2$ cloning process, the UNOT gate and the quantum tomographic characterization of the optimal partial transpose map of polarization encoded qubits.
\end{abstract}

\section{INTRODUCTION}

At a fundamental level quantum information (QI) consists of the set of rules that identify and characterize the physical transformations that are applicable to the quantum state of any information system. Because of the constraints established by the quantum rules it is found that several classical information tasks are forbidden or cannot be perfectly extended to the quantum world. A well known and relevant QI limitation consists of the impossibility of perfectly cloning (copying) any unknown qubit $|\phi\rangle[1]$. In other words, the map $|\phi\rangle \stackrel{U}{\rightarrow}|\phi\rangle|\phi\rangle$ cannot be realized by Nature because it does not belong to the set of Completely Positive $(C P)$ maps, i.e. the only ones consistent with all requirements of quantum mechanics [2]. This basic result may be the most fundamental difference between classical and quantum information theory. Another forbidden operation is the NOT gate that maps any $|\phi\rangle$ in its orthogonal state $\left|\phi^{\perp}\right\rangle[3]$. Even if these two processes are unrealizable in their exact forms, they can be optimally approximated by the so-called universal quantum machines, i.e. which exhibit the minimum possible noise. A better understanding of these devices is important since the exact characterization of the quantum constraints within basically simple QI processes is useful to design more sophisticated algorithms and protocols and to assess the limit performance of complex networks, such as a quantum computer. In this paper we shall analyze the optimal realizations of the NOT gate and of the cloning machine within the enlightening perspective suggested by the new linear optical method that has been recently adopted to achieve such realizations.

The efficiency of a gate, i.e. that measures how close its action is to the desired one, is generally quantified by the fidelity $\mathcal{F} . \mathcal{F}=1$ implies a perfect implementation, while noisy processes correspond to $\mathcal{F}<1$. The Universal NOT (UNOT) gate, the optimal approximation of the NOT gate, maps $N$ identical input qubits $|\phi\rangle$ into $M$ optimal flipped ones in the state $\sigma_{\text {out }}$. It achieves the fidelity : $\mathcal{F}_{N \rightarrow M}^{*}\left(\left|\phi^{\perp}\right\rangle, \sigma_{\text {out }}\right)=\left\langle\phi^{\perp}\left|\sigma_{\text {out }}\right| \phi^{\perp}\right\rangle$ $=(N+1) /(N+2)$ that depends only on the number of the input qubits [4]. Indeed the fidelity of the UNOT gate is exactly the same as the optimal quantum estimation fidelity [5]. This means that such process may be modelled as a "classical", i.e. exact, preparation of $M$ identical flipped qubits following the quantum, i.e. inexact, estimation of $N$ input states. Only this last operation is affected by noise. Only in the limit $N \rightarrow \infty$ a perfect estimation of the input state is achieved and a perfect flipping operation is also realized. Differently from the UNOT gate, the Universal Optimal Quantum Cloning Machine (UOQCM), which transforms $N$ identical qubits $|\phi\rangle$ into $M$ identical copies $\rho_{\text {out }}$, achieves as optimal fidelity : $\mathcal{F}_{N \rightarrow M}\left(|\phi\rangle, \rho_{\text {out }}\right)=\left\langle\phi\left|\rho_{\text {out }}\right| \phi\right\rangle=$ $(N M+M+N) /(M N+2 M)=(N+1+\beta) /(N+2)$ with $\beta \equiv N / M \leq 1 \quad[6-8]$. As we can see $\mathcal{F}_{N \rightarrow M}\left(|\phi\rangle, \rho_{\text {out }}\right)$ is larger than the one obtained by the $N$ estimation approach and reduces to that result for $\beta \rightarrow 0$, i.e. for an infinite number of copies. Of course the zero-cloning condition is expressed by $\beta=1$ and $\mathcal{F}_{N \rightarrow N}=1$. The extra positive term $\beta$ in the above expression accounts for the excess of quantum information which, originally stored in $N$ states, is optimally redistributed by entanglement among the $M-N$ remaining blank qubits encoded by UOQCM [9]. Precisely, the entanglement is established by the cloning process between the blank qubits and the machine itself which may be modelled as a "ancilla" information system.

The first conceptual approach to the realization of 
these transformations is based on finding a suitable unitary operator $U_{N M}$, deterministically realized by means of a quantum network and acting on $N$ input qubits and on $2(M-N)$ ancillary qubits. At the output of this device we obtain $M$ and $(M-N)$ qubits which are, respectively, the optimal clones and the best flipped qubits of the input ones [10]. A different approach to the probabilistic implementation of the $N \rightarrow M$ cloning process has been proposed by Werner [11]. It is based on the action of a projective operation on the symmetric subspace of the $N$ input qubits and $(M-N)$ blank ancillas. This transformation assures the uniform distribution of the initial information into the overall system and guarantees that all output qubits are indistinguishable. While previous realizations [12-16] were inspired by the first approach, the work reported in the present paper follows the path established by the last theoretical proposals, as we see shortly.

In Quantum Optics let the qubit to be codified by the polarization state of a single photon. Precisely in this context it was proposed to realize the $U_{N M}$ transformation by exploiting any amplification process e.g. realized by the Quantum Injected Optical Parametric Amplifier (QIOPA) in the entangled configuration $[17,18]$. Indeed the experimental demonstrations of the UOQCM and the UNOT gate have been reported by exploiting precisely this technique [12-14]. The cloning process (but not the UNOT gate) was also realized by a simple laser amplifier in a $E r^{3+}-$ doped optical fiber [15].

In the present work the more direct qubit symmetrization path proposed by Werner was taken, as said. In this perspective an entirely new scenario has been disclosed by the recent discovery that it is possible to implement contextually the $1 \rightarrow 2$ universal quantum cloning machine (UOQCM) and the $1 \rightarrow 1$ universal NOT gate by slightly modifying the Quantum State Teleportation $(Q S T)$ protocol [19]. Since in this case the UNOT gate is transferred, i.e. teleported, in a different location, it will be referred to as the Tele-UNOT gate. This indeed realizes a novel QI protocol: the "teleportation of a quantum operation".

In Section II of the present work the Tele-UNOT protocol is investigated theoretically by a quantum network approach on the basis of the qubit symmetrization process. Furthermore, this same process is shown to lead very naturally to the efficient qubit purification protocol recently realized experimentally [20,21]. Section III reports a most general application of the symmetrization protocol, indeed one of the key results of the present work: the programmable optimal teleportation of any anti-unitary gate acting on qubits. In Section IV a general and comprehensive theory of the $N \rightarrow M$ UOQCM and of the $N \rightarrow(M-N)$ Tele-UNOT gate is given by adopting a novel and straightforward theoretical approach to the problem, i.e. the well established $\left|J, J_{z}\right\rangle$ angular momentum formalism of a general $J$-spin system. A detailed account of the experimental realization of UOQCM and Tele-UNOT with polarization encoded qubits by two alternative approaches is reported in Section V [19,22]. In Section VI the experimental implementation and characterization of the optimal transpose of a $2 \times 2$ density operator by a stochastic method is reported. This is done in order to investigate, at a deeper level how the UNOT gate can be realized starting from the teleportation protocol. Finally, in Section VII the main results of the work are summarized and considered in the perspectives of modern quantum information, computation and estimation theories.

\section{QUBIT SYMMETRIZATION}

The protocol that realizes the $1 \rightarrow 2$ UOQCM and $1 \rightarrow 1$ Tele-UNOT gate, involves two distant partners: Alice $(\mathcal{A})$ and $\operatorname{Bob}(\mathcal{B})$. $\mathcal{A}$ holds the unknown input qubit $S$ in a generic state $|\phi\rangle_{S}$, while $\mathcal{B}$ shall finally receive this qubit encoded optimally by the UNOT transformation of $|\phi\rangle_{S}$. Let $\mathcal{A}$ and $\mathcal{B}$ share the entangled singlet state of two qubits $A, B:\left|\Psi^{-}\right\rangle_{A B}=2^{-\frac{1}{2}}\left(|\phi\rangle_{A}\left|\phi^{\perp}\right\rangle_{B}-\left|\phi^{\perp}\right\rangle_{A}|\phi\rangle_{B}\right)$, as in the QST protocol [23]. The adoption of the singlet state throughout this work guarantees, in virtue of its $\mathrm{SU}(2)$ invariance, the universality of the overall process. The overall state of the system $\left|\Omega_{S A B}\right\rangle$ reads:

$$
\left|\Omega_{S A B}\right\rangle=2^{-\frac{1}{2}}|\phi\rangle_{S}\left(|\phi\rangle_{A}\left|\phi^{\perp}\right\rangle_{B}-\left|\phi^{\perp}\right\rangle_{A}|\phi\rangle_{B}\right)
$$

Let $\mathcal{A}$ to apply to the overall initial state $\left|\Omega_{S A B}\right\rangle$ the projective operator $P_{S A}$ over the symmetric subspace of the qubits $S$ and $A$ :

$$
P_{S A}=\left(\mathbb{I}_{S A}-\left|\Psi^{-}\right\rangle_{S A}\left\langle\left.\Psi^{-}\right|_{S A}\right)\right.
$$

The projection is successfully realized with probability $p=\frac{3}{4}$. In this case the normalized output state is

$$
\begin{gathered}
\left|\Xi_{S A B}\right\rangle= \\
=\sqrt{\frac{2}{3}}|\phi\rangle_{S}|\phi\rangle_{A}\left|\phi^{\perp}\right\rangle_{B^{-}} \sqrt{\frac{1}{6}}\left(|\phi\rangle_{S}\left|\phi^{\perp}\right\rangle_{A}+\left|\phi^{\perp}\right\rangle_{S}|\phi\rangle_{A}\right)|\phi\rangle_{B}
\end{gathered}
$$

A one bit of classical communications sent by $\mathcal{A}$ announces to $\mathcal{B}$ the success of the symmetrization protocol. This one leaves the two qubits $S, A$ held by $\mathcal{A}$ in the same mixed state

$$
\rho_{S}=\rho_{A}=\frac{5}{6}|\phi\rangle\left\langle\phi\left|+\frac{1}{6}\right| \phi^{\perp}\right\rangle\left\langle\phi^{\perp}\right|
$$

which represent the optimal output of the $1 \rightarrow 2$ cloning process for the input state $|\phi\rangle_{S}$ with the expected fidelity 
$: \mathcal{F}_{1 \rightarrow 2}=\frac{5}{6}$. Contextually, the qubit $B$ held by $\mathcal{B}$ is left by the protocol in the mixed state

$$
\rho_{B}=\frac{1}{3}|\phi\rangle\left\langle\phi\left|+\frac{2}{3}\right| \phi^{\perp}\right\rangle\left\langle\phi^{\perp}\right|
$$

and again the corresponding fidelity of the $1 \rightarrow 1$ optimal Tele-NOT process is the expected one: $\mathcal{F}_{1 \rightarrow 1}^{*}=\frac{2}{3}$.

Note that the presence of the entangled state $\left|\Psi^{-}\right\rangle_{A B}$ is not strictly necessary for the sole implementation of the quantum cloning process as, for this purpose we could apply $P_{S A}$ to an initial state $|\phi\rangle_{S}\left\langle\left.\phi\right|_{S} \otimes \frac{I_{A}}{2}\right.$ as shown experimentally by [22].

\section{A. Implementation of the projection by a quantum network}

The projector $P_{S A}(2)$ into the symmetric space can be implemented by means of a quantum circuit, in analogy with the protocol devised for QST [22]. The projection is obtained by combining Hadamard gates, $C-N O T$ gates, a Toffoli gate and the projective measurement of an ancilla qubit $\widetilde{a}$, initially in the state $|0\rangle_{\widetilde{a}}$ (Fig.2). Let us analyze here in more details the logic of this network. The box "EPR preparation" prepares the singlet state $\left|\Psi^{-}\right\rangle_{A B}$ starting from the qubits $|1\rangle_{A}$ and $|1\rangle_{B}$. Hence the state of the overall system is

$$
\begin{gathered}
|\phi\rangle_{S} \otimes\left|\Psi^{-}\right\rangle_{A B} \otimes|0\rangle_{\widetilde{a}}= \\
=\frac{1}{2}\left[\begin{array}{c}
-\left|\Psi^{-}\right\rangle_{S A}|\phi\rangle_{B}-\left|\Psi^{+}\right\rangle_{S A} \sigma_{Z}|\phi\rangle_{B}+ \\
\left|\Phi^{-}\right\rangle_{S A} \sigma_{X}|\phi\rangle_{B}+\left|\Phi^{+}\right\rangle_{S A} \sigma_{Z} \sigma_{X}|\phi\rangle_{B}
\end{array}\right] \otimes|0\rangle_{\widetilde{a}}
\end{gathered}
$$

The box labelled (1) transforms the state $\left|\Psi^{-}\right\rangle_{S A}$ into $|1\rangle_{S}|1\rangle_{A}$, while the other three Bell states $\left\{\left|\Psi^{+}\right\rangle_{S A},\left|\Phi^{-}\right\rangle_{S A},\left|\Phi^{+}\right\rangle_{S A}\right\}$ are respectively transformed into $\left\{|0\rangle_{S}|1\rangle_{A},|1\rangle_{S}|0\rangle_{A},|0\rangle_{S}|0\rangle_{A}\right\}$. By means of a Toffoli gate [24], the state $|1\rangle_{S}|1\rangle_{A}$ induces the flipping of the qubit $\widetilde{a}$ from $|0\rangle_{\widetilde{a}}$ to $|1\rangle_{\widetilde{a}}$, whereas the other states leave the qubit $\widetilde{a}$ unaltered. The state after the Toffoli gate operation reads

$$
\frac{1}{2}\left[\begin{array}{c}
-|1\rangle_{S}|1\rangle_{A}|\phi\rangle_{B}|1\rangle_{\widetilde{a}}-|0\rangle_{S}|1\rangle_{A} \sigma_{Z}|\phi\rangle_{B}|0\rangle_{\widetilde{a}}+ \\
|1\rangle_{S}|0\rangle_{A} \sigma_{X}|\phi\rangle_{B}|0\rangle_{\widetilde{a}}+|0\rangle_{S}|0\rangle_{A} \sigma_{Z} \sigma_{X}|\phi\rangle_{B}|0\rangle_{\widetilde{a}}
\end{array}\right](7)
$$

Finally the action of the box labelled (2) restores the initial states of the qubits $S$ and $A$ leading to $|\Sigma\rangle_{S A B \widetilde{a}}$ equal to:

$$
\frac{1}{2}\left[\begin{array}{c}
-\left|\Psi^{-}\right\rangle_{S A}|\phi\rangle_{B}|1\rangle_{\widetilde{a}}-\left|\Psi^{+}\right\rangle_{S A} \sigma_{Z}|\phi\rangle_{B}|0\rangle_{\widetilde{a}}+ \\
\left|\Phi^{-}\right\rangle_{S A} \sigma_{X}|\phi\rangle_{B}|0\rangle_{\widetilde{a}}+\left|\Phi^{+}\right\rangle_{S A} \sigma_{Z} \sigma_{X}|\phi\rangle_{B}|0\rangle_{\widetilde{a}}
\end{array}\right]
$$

If the projective measurement on the ancilla qubit $\widetilde{a}$ gives as result "1" the qubits $A$ and $S$ end up in the state
$\left|\Psi^{-}\right\rangle_{S A}$ while the qubit $B$ is in the state $|\phi\rangle$, which has been then teleported from $\mathcal{A}$ to $\mathcal{B}$. If we obtain the result "0" the overall state becomes equal to $\left|\Xi_{S A B}\right\rangle$ (3). The result of the ancilla measurement is communicated to Bob and we realize the optimal quantum cloning machine and the Tele-UNOT gate of the input qubit $|\phi\rangle$.

The circuit proposed by Brassard [25] to model QST, and then realized by NMR techniques [26] achieves teleportation by means of single qubit gates and $C-N O T$ gates. The present scheme somewhat retraces the path of that circuit but there an ancilla state and a Toffoli gate replace the Bell-measurement device with the detection of the realization of state-symmetrization by the measurement apparatus at the site $\mathcal{A}$. Moreover, the circuit proposed by Bužek et al. [10] and realized adopting NMR technique [16], to model the quantum cloning and the UNOT gate in the conventional devices, e.g. in a QIOPA system [14], differs from the present one since there QST is not considered explicitly.

\section{B. Purification of single qubits.}

The circuit above represents a versatile tool for physically implementing several relevant QI processes based on the state symmetrization process. For instance it can be adopted to implement the optimal quantum purification of two qubits according to the symmetrization scheme proposed by Cirac et al. [20]. This one addresses the issue of the purification of $N$ equally prepared qubits in the mixed state $\rho=\xi|\phi\rangle\langle\phi|+\frac{1}{2}(1-\xi) \mathbb{I}$, where $0 \leq \xi \leq 1$. The procedure allows to distill out of a set of mixed states a subset of states with a higher degree of purity, i.e. the state purity is enhanced by filtering out some amount of the noise. The purification scheme for $N=2$, consisting of a projection of two polarization $(\pi)$ encoded qubits onto the symmetric subspace, can be implemented by means of a symmetric beam-splitter $(B S)$, was recently reported by [21]. It can be easily checked that this purification protocol can be modelled by the quantum network of Fig.2.

In order to further enlighten the connection between the purification and cloning processes, let us consider the action of the symmetric projector on two non entangled qubits having the same orientation on the Bloch sphere but, generally, with different degree of mixedness:

$$
\begin{aligned}
& \rho_{S}=\frac{1+\lambda_{S}}{2}|\phi\rangle\left\langle\phi\left|+\frac{1-\lambda_{S}}{2}\right| \phi^{\perp}\right\rangle\left\langle\phi^{\perp}\right| \\
& \rho_{A}=\frac{1+\lambda_{A}}{2}|\phi\rangle\left\langle\phi\left|+\frac{1-\lambda_{A}}{2}\right| \phi^{\perp}\right\rangle\left\langle\phi^{\perp}\right|
\end{aligned}
$$

We apply the projector $P_{S A}$ to the overall system $\rho_{S} \otimes$ $\rho_{A}$. The success probability of the procedure is equal 
to $p=\frac{1}{4}\left(3+\lambda_{A} * \lambda_{S}\right)$. Let us introduce a parameter $\Delta=\frac{1}{2}\left(\lambda_{A}+\lambda_{S}\right)$ quantifying the information over the input qubits. The mean fidelity $\mathcal{F}_{\text {in }}$ of the input qubits is found: $\mathcal{F}_{\text {in }} \equiv \frac{1}{2}\left(\left\langle\phi\left|\rho_{S}\right| \phi\right\rangle+\left\langle\phi\left|\rho_{A}\right| \phi\right\rangle\right)=\frac{1}{2}(1+\Delta)$. After the projection the output qubits, which are equal since they belong to the symmetric subspace, are

$$
\rho_{\text {out }}=\frac{1+\lambda_{\text {out }}}{2}|\phi\rangle\left\langle\phi\left|+\frac{1-\lambda_{\text {out }}}{2}\right| \phi^{\perp}\right\rangle\left\langle\phi^{\perp}\right|
$$

with $\lambda_{\text {out }}=\frac{\Delta}{p}$. The fidelity of the output qubits reads

$$
\mathcal{F}_{\text {out }}\left(\rho_{\text {out }},|\phi\rangle\right)=\frac{1}{2}\left(1+\Delta p^{-1}\right)
$$

For $\lambda_{S}=1$ and $\lambda_{A}=0, p=\frac{3}{4}, \mathcal{F}_{i n}=3 / 4$ and $\mathcal{F}_{\text {out }}=5 / 6$. These values indeed correspond to the optimal quantum cloning based on symmetrization [22]. For the case of the qubit purification protocol, $\lambda_{S}=\lambda_{A}=\lambda$ the following relations hold: $p=\frac{1}{4}\left(3+\lambda^{2}\right), \mathcal{F}_{\text {in }}=\frac{1}{2}(1+\lambda)$ and $\mathcal{F}_{\text {out }}=\frac{1}{2}\left(1+\lambda p^{-1}\right)$.

\section{PROGRAMMABLE OPTIMAL TELEPORTATION OF ANY ANTI-UNITARY MAP.}

As a consequence of the complete positivity (CP) character of any realizable physical map [24], any antiunitary transformation cannot be implemented with fidelity $\mathcal{F}=1$. Any general anti-unitary operator $A$ can be expressed as $A=U^{A} K$, where $U^{A}$ is a unitary operator depending on $A$ and $K$ is the complex-conjugate operator that transforms any coefficient multiplying a ket standing at the right of $K$ into its complex conjugate [27]. $K$ implements the transpose map of the density matrix $\rho: \mathcal{E}_{\text {trans }}(\rho) \equiv \rho^{T}$. For instance the NOT operator is: $A^{N O T}=\sigma_{Y} K$, where $\sigma_{Y}$ is a Pauli operator. We can then express $A$ as:

$$
A=U^{A} \sigma_{Y} A^{N O T}
$$

Precisely, let to express the given "impossible" antiunitary processes as: $A|\phi\rangle=\left|\phi^{A}\right\rangle$ and $A^{N O T}|\phi\rangle=\left|\phi^{\perp}\right\rangle$. In the previous Sections we have seen how to implement the optimal approximation of the NOT operator with fidelity $\mathcal{F}^{*}=\frac{2}{3}$. Such transformation is performed by the map: $\mathcal{E}_{U N O T}(|\phi\rangle\langle\phi|)=\frac{2}{3}\left|\phi^{\perp}\right\rangle\left\langle\phi^{\perp}\left|+\frac{1}{3}\right| \phi\right\rangle\langle\phi|$. We may now ask what is the value of $\mathcal{F}^{*}$ of the optimal approximation to the general anti-unitary operator $A$. It is easy to show that such value is again $\mathcal{F}_{A}=\left\langle\phi^{A}\left|\rho_{\text {out }}\right| \phi^{A}\right\rangle=\frac{2}{3}$, the same as for the U-NOT one. Indeed, consider the action over the input density matrix $\rho=|\phi\rangle\langle\phi|$ of the map
$\mathcal{E}_{A}(\rho)$. In virtue of Eq. (13) is $A\left(A^{N O T}\right)^{-1}=U^{A} \sigma_{Y}$ and then

$$
\begin{gathered}
\mathcal{E}_{A}(\rho)=U^{A} \sigma_{Y} \mathcal{E}_{U N O T}(\rho) \sigma_{Y} U^{A+}= \\
=\frac{2}{3}\left|\phi^{A}\right\rangle\left\langle\phi^{A}\left|+\frac{1}{3}\right| \phi^{A \perp}\right\rangle\left\langle\phi^{A \perp}\right|
\end{gathered}
$$

leading to $\mathcal{F}_{A}=\frac{2}{3}$. If we could approximates $A$ with a higher fidelity, then we could also implement a UNOT gate with fidelity $\mathcal{F}>\frac{2}{3}$, which is impossible. We can then assert that the maximum fidelity achievable in a optimal universal approximation to any general anti-unitary transformation applied to one qubit is $\mathcal{F}=\frac{2}{3}$. In other words, this last $\mathcal{F}$ value may be thought to establish a class of one qubits anti-unitary maps.

Let us generalize the above concepts in the framework of the QST protocol. Note first that, by exploiting the result of Eq.(14) any optimal anti-unitary operation can be teleported by adopting a different entangled state in the protocol described in Section II. Let Alice and Bob share the maximally entangled state obtained by any local transformation of the singlet: $|\Psi\rangle_{A B}=\left(\mathbb{I}_{A} \otimes U_{B}^{\dagger}\right)\left|\Psi^{-}\right\rangle_{A B}=\left(U_{A} \otimes \mathbb{I}_{B}\right)\left|\Psi^{-}\right\rangle_{A B}$ where $U=\exp (-i \phi \sigma \bullet \mathbf{n}) / 2=[\cos (\phi / 2) \mathbb{I}-i \sin (\phi / 2) \sigma \bullet \mathbf{n}]$ is a general unitary that can be applied either at the Alice's or at the Bob's sites. Let us discuss here the first option, the most interesting one, by referring again to the Tele-UNOT protocol [19]. After projection into the symmetric subspace, Alice detects the two optimal clones of the input qubit $\rho_{S}$ while, conditionally, Bob detects the qubit $\rho_{B}=U^{\dagger} \mathcal{E}_{U N O T}\left(\rho_{S}\right) U$, i.e. resulting from the application to $\rho_{S}$ of the optimal approximation of the anti-unitary operator $U^{\dagger} \sigma_{Y} K$. The choice of $U$, applied in the Alice's site (or, alternatively the one of $U^{\dagger}$ applied at Bob's site) establishes the class of all anti-unitary operators $A$ to be teleported with fidelity $\mathcal{F}_{A}=\frac{2}{3}$. For instance, according to the discussion above, $U=\mathbb{I}$, i.e. $\phi=0$, leads to the optimal Tele $-U N O T$ gate, while $U=$ $\sigma_{Y}$, i.e. $\phi=\pi / 2$ and $\sigma \bullet \mathbf{n}=\sigma_{Y}$, leads to the optimal Tele - Transposition gate [28], etc. It is quite remarkable that, according to the QST concept, both the input state $\rho_{S}$ and the operator $U$ are realized and kept under control at the Alice's site while the optimal anti-unitary gate is transferred far apart by the noiseless non-local channel. This novel universal and optimal "programmable gate-teleportation" process is represented in Fig. 1 by the insertion in the logical circuit of the general unitary $U$. It may considered as a relevant realization of the universal quantum processor reported by [29]. 


\section{ANGULAR MOMENTUM THEORY OF THE $N \rightarrow M$ UNIVERSAL OPTIMAL CLONING AND OF THE $N \rightarrow(M-N)$ U-NOT GATE.}

So far the quantum cloning of $N^{\prime}=1$ qubit into $M^{\prime}=2$ qubits and the optimal flipping of $N^{\prime}=1$ qubit into $\left(M^{\prime}-N^{\prime}\right)=1$ qubit have been considered. By generalizing the Tele-UNOT protocol to $N>1$ identical input qubits and $(M-N)>1$ entangled pairs we obtain the teleportation of $(M-N)$ qubits optimally flipped at Bob's site and, contextually, the optimal realization at Alice's site of the $N \rightarrow M$ cloning. Let us briefly describe this protocol: $N$ input qubits in the state $|\phi\rangle=\alpha|0\rangle+\beta|1\rangle=U_{\phi}|0\rangle$ are sent to Alice who shares with Bob $(M-N)$ entangled pairs, all in the singlet state to guarantee the universality of the process: $\left|\Psi^{-}\right\rangle=2^{-1 / 2}(|0\rangle|1\rangle-|1\rangle|0\rangle)$. The initial state of the overall system is $|\Omega\rangle=|\phi\rangle^{\otimes N}\left|\Psi^{-}\right\rangle^{\otimes(M-N)}=$ $U_{\phi}^{\otimes N}|0\rangle^{\otimes N}\left|\Psi^{-}\right\rangle^{\otimes(M-N)}$. Alice applies the projector $P_{\text {sym }}^{M}$ over the symmetric subspace to her $M$ qubits, i.e. $N$ input qubits $+(M-N)$ ancilla qubits, and communicates to Bob the positive realization of the symmetrization procedure by means of one classical bit. The overall input-output protocol is enlightened by the scheme reported in Fig. 3.

In order to simplify the demonstration of the optimality, we exploit the universality of the projection procedure. Indeed:

$$
\begin{aligned}
& \left(P_{\text {sym }}^{M} \otimes \mathbb{I}_{B}\right)\left[U_{\phi}^{\otimes N}|0\rangle^{\otimes N}\left|\Psi^{-}\right\rangle^{\otimes(M-N)}\right]= \\
= & U_{\phi}^{\otimes(2 M-N)} P_{\text {sym }}^{M} \otimes \mathbb{I}_{B}|0\rangle^{\otimes N}\left|\Psi^{-}\right\rangle^{\otimes(M-N)}
\end{aligned}
$$

for any $U_{\phi} \in S U(2) . \mathbb{I}_{B}$ is the identity operator acting on the Hilbert space of Bob's qubits. The covariance property expressed in (15) is assured by the invariance of the singlet state for simultaneous unitary operations on the two qubits $\left|\Psi^{-}\right\rangle=U_{\phi}^{\otimes 2}\left|\Psi^{-}\right\rangle$and by the commutation property of the projector $P_{s y m}^{M}:\left[P_{s y m}^{M}, U^{\otimes M}\right]=0$. The covariance property allows us to assume as input state $|\phi\rangle=|0\rangle$ without lack of generality.

In the following part of this Section a very general and comprehensive theory of the universal optimal cloning and U-NOT gate is given by adopting, in a straightforward fashion, the well established $\left|J, J_{z}\right\rangle$ angular momentum formalism of a general $J$-spin system [30]. The overall symmetric state of the $N$ input qubits is taken to corresponds to a system with total spin $\frac{N}{2}:|0\rangle^{\otimes N} \doteq\left|\frac{1}{2}, \frac{1}{2}\right\rangle^{\otimes N}=\left|\frac{N}{2} ; \frac{N}{2}\right\rangle$. Accordingly, the joint state of the $N$ input qubits and of $(M-N)$ entangled pairs $|\Omega\rangle$ is re-expressed in the spin formalism as $\left|\frac{N}{2} ; \frac{N}{2}\right\rangle \otimes|0,0\rangle^{\otimes M-N}=\left|\frac{N}{2} ; \frac{N}{2}\right\rangle$ because the $M-N$ singlets contribute to the total spin with $J=J_{z}=0$. The symmetrization projector $P_{s y m}^{M}$ is defined as: $P_{s y m}^{M}=\sum_{k=0}^{M}\left|\frac{M}{2} ; \frac{M}{2}-k\right\rangle\left\langle\frac{M}{2} ; \frac{M}{2}-k\right|$ because all symmetrized "cloned" spins are equally directed in the Poincare' space and the projection is over the maximum allowed value of $J$. After the action of $P_{s y m}^{M}$ we obtain the following normalized output state

$$
\begin{gathered}
\left|\Omega^{\prime}\right\rangle=\frac{P_{\text {sym }}^{M} \otimes \mathbb{I}_{B}\left|\frac{N}{2} ; \frac{N}{2}\right\rangle}{\left.\left|P_{\text {sym }}^{M} \otimes \mathbb{I}_{B}\right| \frac{N}{2} ; \frac{N}{2}\right\rangle \mid}= \\
\sum_{k=0}^{M-N} b_{k}\left|\frac{M}{2} ; \frac{M}{2}-k\right\rangle_{A} \otimes\left|\frac{M-N}{2} ; \frac{-(M-N)}{2}+k\right\rangle_{B}
\end{gathered}
$$

where $b_{k}=(-1)^{k} \sqrt{\frac{N+1}{M+1}} \sqrt{\frac{(M-N) !(M-k) !}{M !(M-N-k) !}}$ is the Clebsch - Gordan coefficient $\left\langle j_{1} ; m_{1 k} ; j_{2} ; m_{2 k} \mid j_{1} ; j_{2} ; j_{T O T} ; m_{T O T}\right\rangle$ with $j_{1}=\frac{M}{2}, j_{2}=\frac{M-N}{2}, m_{1 k}=\frac{M}{2}-k, m_{2 k}=$ $\frac{-(M-N)}{2}+k, j_{T O T}=\frac{N}{2}, m_{T O T}=\frac{N}{2}([30]$, Ch.3.6) In the above representation, the overall output state of the cloner is written as the composition of two angular momenta: $\mathbf{J}_{C}, \mathbf{J}_{A C}$ defined respectively over the "cloning" and "anticloning" output channels. In the present context, these angular momenta correspond to the output states realized at the Alice's and Bob's sites respectively. The success probability of the procedure is $\left.\left|P_{s y m}^{M} \otimes \mathbb{I}_{B}\right| \frac{N}{2} ; \frac{N}{2}\right\rangle\left.\right|^{2}=\frac{1}{2^{M-N}} \frac{1+M}{1+N}$. We note that the $(M-N)$ Bob's qubits assume the maximum allowed value of $J=\frac{M-N}{2}$, thus they lie in the symmetric subspace in analogy with the Alice's ones.

The fidelities of the cloning and of the UNOT processes can be inferred re-arranging the output state (16) as follow

$$
\begin{gathered}
\left|\Omega^{\prime}\right\rangle= \\
\sum_{k=0}^{M-N} b_{k}\left|\left\{(M-k) \phi ; k \phi^{\perp}\right\}\right\rangle_{A} \otimes\left|\left\{k \phi ;(M-N-k) \phi^{\perp}\right\}\right\rangle_{B}
\end{gathered}
$$

The notation $\left|\left\{p \phi ; q \phi^{\perp}\right\}\right\rangle$ stands for a total symmetric combination of $p$ qubits in the state $|\phi\rangle$ and of $q$ qubits in the state $\left|\phi^{\perp}\right\rangle$. All the $(p+q)$ qubits belonging in such state have an identical reduced density matrix equal to

$$
\rho_{p, q}=\frac{p}{p+q}|\phi\rangle\left\langle\phi\left|+\frac{q}{p+q}\right| \phi^{\perp}\right\rangle\left\langle\phi^{\perp}\right|
$$

The fidelity $\mathcal{F}_{C L O N}$ of the cloning process is thus

$$
\begin{aligned}
\mathcal{F}_{N \rightarrow M} & =\sum_{k=0}^{M-N}\left|b_{k}\right|^{2}\left\langle\phi\left|\rho_{M-k, k}\right| \phi\right\rangle= \\
& =\sum_{k=0}^{M-N}\left|b_{k}\right|^{2} \mathcal{F}_{C L O N}^{k}=\frac{N+1+\beta}{N+2}
\end{aligned}
$$


where $\beta=\frac{N}{M}$ and $\mathcal{F}_{C L O N}^{k}=\frac{M-k}{M}$ is the fidelity of the $k$-th term of the summation derived from the expression (18). The above expression of $\mathcal{F}_{N \rightarrow M}$ coincides with the one given in literature [9] and in Sect.I, above. For the UNOT process we obtain

$$
\begin{aligned}
\mathcal{F}_{N \rightarrow(M-N)}^{*} & =\sum_{k=0}^{M-N}\left|b_{k}\right|^{2}\left\langle\phi^{\perp}\left|\rho_{k, M-N-k}\right| \phi^{\perp}\right\rangle= \\
& =\sum_{k=0}^{M-N}\left|b_{k}\right|^{2} \mathcal{F}_{U N O T}^{k}=\frac{N+1}{N+2}
\end{aligned}
$$

where $\mathcal{F}_{U N O T}^{k}=\frac{M-N-k}{M-N}$. This value coincides with the optimal fidelity given by Gisin and Massar [6] of any general measurement of $N$ equal and unknown qubits. Thus we retrieve the result by which, as far as fidelity is concerned, the U-NOT process is equivalent to a quantum estimation measurement followed by a "classical" inversion of the corresponding outcomes [12]. However, as already noted in Sect.I, this is not the case for quantum cloning where the extra term $\propto \beta$ in Eq.19 accounts for the residual information stored in the entanglement of the output "clones".

In analogy with the $1 \rightarrow 2$ UOQCM protocol analyzed in Sect.II, we note once again that the entangled source is not strictly necessary in order to achieve solely the $N \rightarrow M$ cloning process as for this purpose only $(M-N)$ ancilla qubits in a fully mixed state are needed [11]. Furthermore, as a further generalization of the results of Sect.III above, by starting with pairs bearing a different entanglement structure it is possible to teleport a generic, optimal anti-unitary transformation. For instance, the adoption of the triplet state $\left|\Phi^{+}\right\rangle=2^{-1 / 2}(|0\rangle|0\rangle+|1\rangle|1\rangle)$ leads to the quantum cloning machine given by Gisin and Massar [6] by which the $(M-N)$ qubits teleported to Bob represent the optimally transposed transformation of the input qubits. More about this process is reported in Section VI, below.

\section{EXPERIMENTAL OPTICAL IMPLEMENTATIONS.}

In the experiments reported in [19] and [22], the input qubit was codified into the polarization $(\pi)$ state of a single photon belonging to the mode $k_{S}:|\phi\rangle_{S}=$ $\alpha|H\rangle_{S}+\beta|V\rangle_{S}$, whereas an entangled pair $\left|\Psi^{-}\right\rangle_{A B}$ of photons $A$ and $B$, was generated on the modes $k_{A}$ and $k_{B}$ by Spontaneous Parametric Down Conversion (SPDC). The projective operation in the space $H=H_{S} \otimes H_{A}$ was realized by exploiting the linear superposition of the modes $k_{S}$ and $k_{A}$ within a $50: 50$ beam-splitter, $B S_{A}$
(Figs.4-6). This superposition allows a partial Bell measurement on the $B S_{A}$ output states which is needed to implement the cloning machine and the Tele-UNOT gate. Consider the overall output state realized on the two modes $k_{1}$ and $k_{2}$ of $B S_{A}$ and expressed by a superposition of the Bell states: $\left\{\left|\Psi^{-}\right\rangle_{S A},\left|\Psi^{+}\right\rangle_{S A},\left|\Phi^{-}\right\rangle_{S A},\left|\Phi^{+}\right\rangle_{S A}\right\}$. The realization of the singlet $\left|\Psi_{S A}^{-}\right\rangle$is identified by the emission of one photon on each output mode of $B S_{A}$, while the realization of the other three Bell states implies the emission of 2 photons either on mode $k_{1}$ or on mode $k_{2}$. This Ou-Mandel interference process, expressing a Bose mode coalescence (BMC) of the two photons over the same mode, was experimentally identified by a coincidence event between two detectors coupled to the output mode $k_{2}$ by means of an additional 50 : 50 beamsplitter. The identical effect expected on mode $k_{1}$ was neglected, for simplicity. As just shown, this condition assured the simultaneous experimental realization of the UNOT and UOQCM processes, here detected by a postselection technique.

Note that, while the present Tele-UNOT protocol with $\pi$-encoded qubits is fully realizable by linear optical methods, the full implementation by these methods of the Bell measurement in the standard QST protocol is impossible [31]. Hyper-entanglement with additional degrees of freedom [32] or a network of C-NOT gates are required to perform that task. In general, by a balanced beam splitter a POVM measurement is able to distinguish between the symmetric and the asymmetric components of the overall state of two qubits. Indeed, the projection into the symmetric space lies at the core of the cloning process, as epitomized by the present work $[11,19,22,33]$. The cloning process was investigated in two independent experiments which enlighten different features of the protocol. The first experiment, involving only two photons generated by the same SPDC process, demonstrated that an entangled state is not necessary for the UOQCM implementation and achieves a fidelity close to the limit value. The second one, which adopts photons belonging to independent sources, experimentally demonstrated the overall process.

\section{A. Ou Mandel Cloning}

In order to clone the input qubit $S$, an entangled state of the qubits $A$ and $B$ was not necessary as only a qubit $A$ in a fully mixed state was needed [22]. Hence we carried out a first experiment involving only two qubits. A pair of photons with equal wavelength (wl) $\lambda=532 \mathrm{~nm}$ and coherence-time $\tau_{c o h}=80 \mathrm{fs}$, was generated by a SPDC process in a Type I BBO crystal in the initial polarization product state $|H\rangle_{S}|H\rangle_{A}$ (Fig.4). The non-linear 
(NL) crystal was pumped by a continuous-wave (cw) UV beam with wl $\lambda=266 \mathrm{~nm}$, created by fourth-harmonics generation in a OPO cavity (Coherent:MBD266) by a cw Nd:YAG laser (Coherent:VERDI) with wl $\lambda=532 \mathrm{~nm}$. This sophisticated system provided a true single mode UV beam with linewidth $<100 \mathrm{MHz}$ and high power, up to $400 \mathrm{~mW}$ allowing a high coincidence rate. The photons $S$ and $A$ from SPDC pairs were injected on the two input modes $k_{S}$ and $k_{A}$ of $B S_{A}$ with an adjustable mutual temporal delay $\Delta t$. The input qubit $|\phi\rangle_{S}$ associated with mode $k_{S}$ was polarization encoded by means of a waveplate (wp) $W P_{S}$. The transformation used to map the state $|H\rangle_{A}$ into $\rho_{A}=\frac{\mathbb{I}_{A}}{2}$ was achieved by stochastically rotating, during each experimental run, a $\lambda / 2 \mathrm{wp}$ $\left(W P_{A}\right)$ inserted on the mode $k_{A}$. In this way the statistical evolution of $|H\rangle_{A}$ into two orthogonal states with equal probability was achieved.

The $\pi$-state on the output mode $k_{2}$ of $B S_{A}$ was analyzed by the combination of the wp $W P_{C}$ and of the polarizing beam splitter (PBS): $P B S_{C}$. For each input $\pi$-state $|\phi\rangle_{S}, W P_{C}$ was set in order to make $P B S_{C}$ to transmit $|\phi\rangle$ and reflect $\left|\phi^{\perp}\right\rangle$. The "cloned" state $|\phi \phi\rangle$ could be detected on mode $k_{2}$ by a two-photon counter, realized in our case by first separating the two photons by an additional $50: 50$ beam splitter $B S_{C}$ and then detecting the coincidence $\left[D_{C}, D_{C}^{\prime}\right]$ between the output detectors $D_{C}$ and $D_{C}^{\prime}$ : Fig. 4. Any coincidence between $D_{C}^{*}$ and $D_{C}$ corresponded to the realization of the state $\left|\phi \phi^{\perp}\right\rangle$. First consider the cloning machine switched off by spoiling the interference of $S$ and $A$ in $B S_{A}$, i.e. by setting: $\Delta t=Z /(2 c)>>\tau_{c o h}$, being $Z$ a micrometrical displacement of $B S_{A}$. In this case, since the states $|\phi \phi\rangle$ and $\left|\phi \phi^{\perp}\right\rangle$ were realized with the same probability on mode $k_{2}$, the rate of coincidences detected by $\left[D_{C}, D_{C}^{\prime}\right]$ were expected to one half of the one detected by $\left[D_{C}^{*}, D_{C}^{\prime}\right]$. By turning on the cloning machine, i.e. by setting $\Delta t \approx 0$ the output density matrices $\rho_{S}, \rho_{A}(4)$ were realized on the mode $k_{2}$ with an enhancement ratio $R=2$ of the counting rate by the set $\left[D_{C}, D_{C}^{\prime}\right]$ and no rate enhancement by $\left[D_{C}^{*}, D_{C}^{\prime}\right]$. All adopted photodetectors $(D)$ were SPCM200 single photon counters and interferential filters with bandwidth (bwth) $\Delta \lambda=5 \mathrm{~nm}$ were placed behind them.

The experimental data are reported in Fig. 4 for three different input $\pi$-states: $|\phi\rangle_{S}=|H\rangle, 2^{-\frac{1}{2}}(|H\rangle+|V\rangle)$, $2^{-\frac{1}{2}}(|H\rangle+i|V\rangle)$. There square and triangular marks refer respectively to the $\left[D_{C}, D_{C}^{\prime}\right]$ and $\left[D_{C}^{\prime}, D_{C}^{*}\right]$ coincidences versus the time setting $Z$. The corresponding experimental values of the cloning fidelity $\mathcal{F}=(2 R+1) /(2 R+2)$ are $\mathcal{F}_{H}=0.827 \pm 0.002, \mathcal{F}_{H+V}=0.825 \pm 0.002, \mathcal{F}_{H+i V}=$ $0.826 \pm 0.002$. These values are in good agreement with the optimal value $\mathcal{F}_{1 \rightarrow 2}=5 / 6=8.333$ corresponding to the limit $S / N$ value: $R=2$. A similar experiment could be performed by adopting a single photon source to produce an ancilla photon on mode $k_{2}$ [34].

\section{B. Cloning + Tele UNOT Gate}

To further investigate the UOQCM, in a second experiment we employed two independent photons generated by uncorrelated processes: the input qubit was obtained by strongly attenuating a coherent beam while the ancilla photon was generated by a SPDC process. In order to observe the UOQCM process the indistinguishability between the two photons at the output mode $k_{2}$ of $B S_{A}$ had to be attained by realizing a single output mode condition with the best possible approximation, as we shall see shortly.

The source of the SPDC process was a Ti:Sa modelocked pulsed laser (Coherent: MIRA) with wl $\lambda=$ $795 \mathrm{~nm}$ and repetition rate $76 \mathrm{MHz}$ (Fig. 5). A weak beam, deflected from the laser beam by a partial reflecting mirror $M$, was strongly attenuated by filters $(A t)$ and delayed by $Z=2 c \Delta t$ via a micrometrically adjustable optical "trombone". This beam was the source of the quasi single-photon state injected into $B S_{A}$ over the mode $k_{S}$. The average number of injected photons was $\bar{n} \simeq 0.1$. Different qubit states $|\phi\rangle_{S}$ were prepared via a $\lambda / 2$ or $\lambda / 4$ wp $W P_{S}$. The UV laser beam with wl $\lambda_{p}=397.5 \mathrm{~nm}$, generated by $2^{\text {nd }}$ - harmonics generation, excited the SPDC source of the singlet $\left|\Psi^{-}\right\rangle_{A B}$. The photons $A$ and $B$ of each entangled pair were emitted over the modes $k_{A}$ and $k_{B}$ with equal wls $\lambda=795 \mathrm{~nm}$. All adopted photodetectors $(D)$ were SPCM-AQR14 single photon counters.

In order to observe the Ou-Mandel interference at the output of $B S_{A}$ a high spatial indistinguishability of the photons $A$ and $S$ was provided by a single mode selector $(M S)$, realized by a $5 \mathrm{~m}$ long single-mode fiber, inserted on mode $k_{2}$ by a fiber coupler Thor Labs $K T 110$. The fixed $\pi$-transformation induced by the propagation inside the fiber was compensated by a Babinet compensator $(B C)$ and a $\lambda / 2 \mathrm{wp}$. The induced rotation was stable up to $1 \%$ for more than 1 day. An interferential filter $(I F)$ with bwth $\Delta \lambda=3 \mathrm{~nm}$ placed in front of each $D$ determined the coherence time of the detected pulses: $\tau_{c o h} \simeq 350 \mathrm{fs}$. Two fixed quartz plates $(Q)$ inserted on the modes $k_{A}$ and $k_{B}$ provided the compensation for the unwanted walk-off effects due to the birefringence of the $\mathrm{BBO}(\beta$-barium borate) nonlinear $(N L)$ crystal providing the source of SPDC.

In order to demonstrate the realization of the linear UOQCM process, the states $\rho_{A}$ and $\rho_{S}$ of the clones $A$ and $S$ at the output of $B S_{A}$ were investigated. According to the quantum analysis given in Sect.II, expressed by Eq.(4), we would expect $\rho_{S}=\rho_{A}=\left(5 \rho_{I N}+\rho_{I N}^{\perp}\right) / 6$ where $\rho_{I N}=|\phi\rangle\langle\phi|$. The measurements were realized 
on the $B S_{A}$ output mode $k_{2}$ by adopting the apparatus shown in Fig. 5. The $\pi$-state on this mode was analyzed by the combination of the wp $W P_{C}$ and of the polarizer beam splitters $P B S$. For each input $\pi$-state $|\phi\rangle_{S}, W P_{C}$ was set in order to make $P B S$ to transmit $|\phi\rangle$ and reflect $\left|\phi^{\perp}\right\rangle$. The "cloned" state $|\phi \phi\rangle$ was detected on mode $k_{2}$ by the coincidence set $\left[\begin{array}{ll}D_{C} & D_{C}^{\prime}\end{array}\right]$. The generation of an entangled pair was tested by detection of one photon on the mode $k_{B}$ by $D_{B}$. Any coincidence detected by the sets $\left[D_{C}, D_{C}^{\prime}, D_{B}\right]$ and $\left[D_{C}, D_{C}^{*}, D_{B}\right]$ implied the realization of the states $|\phi \phi\rangle$ and $\left|\phi \phi^{\perp}\right\rangle$, respectively. The experimental results of the signal-to-noise $(S / N)$ ratio $R$, carried out by coincidence measurements involving $\left[D_{C}, D_{C}^{\prime}, D_{B}\right]$ and $\left[D_{C}, D_{C}^{*}, D_{B}\right]$ are reported in Fig. 5, again for the three different input $\pi$-states: $|\phi\rangle_{S}=|H\rangle$, $|\phi\rangle_{S}=2^{-1 / 2}(|H\rangle+|V\rangle),|\phi\rangle_{S}=2^{-1 / 2}(|H\rangle+i|V\rangle)$. The square and triangular markers there refer respectively to the $\left[D_{C}, D_{C}^{\prime}, D_{B}\right]$ and $\left[D_{C}, D_{C}^{*}, D_{B}\right]$ coincidence plots vs the delay $Z$. The following values of the cloning fidelity $\mathcal{F}=(2 R+1) /(2 R+2)$ were found $\mathcal{F}_{H}=0.821 \pm 0.003$, $\mathcal{F}_{H+V}=0.813 \pm 0.003, \mathcal{F}_{H+i V}=0.812 \pm 0.003$ to be compared with the optimal $\mathcal{F}_{1 \rightarrow 2}=5 / 6 \approx 0.833$ corresponding to the limit $S / N$ value: $R=2$. These results have been evaluated by taking into account the reduction, by a factor $\xi=0.7$, of the $S / N$ ratio $R$ due to unwanted coincidence rates attributable to the spurious simultaneous injection of two photons on the mode $k_{S}$ and to simultaneous emission of two SPDC pairs. The factor $\xi$ was carefully evaluated by a side experiment.

In order to realize the Tele - UNOT protocol, the "Bose coalescence" process was detected on the output mode $k_{2}$ of $B S_{A}$ by the coincidence $\left[\begin{array}{ll}D_{2} & D_{2}^{*}\end{array}\right]$ as shown in Fig. 6. At Bob's site, the polarization state on the mode $k_{B}$ was analyzed by the combination of the wp $W P_{B}$ and of $P B S_{B}$. For each input $\pi-$ state $|\phi\rangle_{S}, W P_{B}$ was set in order to make the $P B S_{B}$ to transmit $|\phi\rangle_{B}$ and to reflect $\left|\phi^{\perp}\right\rangle_{B}$, by then exciting $D_{B}$ and $D_{B}^{*}$ correspondingly. First consider the QST turned off, by setting the optical delay $|Z| \gg c \tau_{c o h}$. In this case, since the states $|\phi\rangle_{B}$ and $\left|\phi^{\perp}\right\rangle_{B}$ were realized with the same probability on mode $k_{B}$, the rate of coincidences detected by the $D$-sets $\left[D_{B}, D_{2}, D_{2}^{*}\right]$ and $\left[D_{B}^{*}, D_{2}, D_{2}^{*}\right]$ were expected to be equal. By turning on the QST, i.e. by setting $|Z|<<c \tau_{c o h}$, the output state $\rho_{B}^{\text {out }}=\left(2 \rho_{I N}^{\perp}+\rho_{I N}\right) / 3$ was realized then implying an enhancement by a factor $R=2$ of the counting rate $\left[D_{B}^{*}, D_{2}, D_{2}^{*}\right]$ and no enhancement of $\left[D_{B}, D_{2}, D_{2}^{*}\right]$. The corresponding 3-coincidence results shown in Fig.6 and involving these 3-detector sets correspond to the injection of three different input $\pi$-states: $|\phi\rangle_{S}=|H\rangle,|\phi\rangle_{S}=2^{-1 / 2}(|H\rangle+|V\rangle)$, $|\phi\rangle_{S}=2^{-1 / 2}(|H\rangle+i|V\rangle)$. As such these results indeed demonstrate the universality of the Tele-UNOT process. In Fig. 6 the square and triangular markers refer respectively to the $\left[D_{B}^{*}, D_{2}, D_{2}^{*}\right]$ and $\left[D_{B}, D_{2}, D_{2}^{*}\right]$ coinci- dences versus the delay $\Delta t$. The Tele $-U N O T$ process was found only to affect the $\left|\phi^{\perp}\right\rangle_{B}$ component, as expected. The $S / N$ ratio $R$ was determined for each resonance curve as the ratio between the values of the resonance peak, i.e. for $Z \simeq 0$, and the no enhancement value, i.e. for $|Z| \approx 0$. The experimental values of the UNOT $f i$ delity $\mathcal{F}^{*}=R /(R+1)$ were found, in correspondence with the three injected $\pi$-states $|\phi\rangle_{S}: \mathcal{F}_{H}=0.641 \pm 0.005$, $\mathcal{F}_{H+V}=0.632 \pm 0.006, \mathcal{F}_{H+i V}=0.619 \pm 0.006$ to be compared with $\mathcal{F}_{1 \rightarrow 1}^{*}=R /(R+1)=2 / 3=0.667$. As for the cloning experiment, the measured correcting factor $\xi=0.7$ has been used to evaluate of the value of the fidelity.

\section{STOCHASTIC EXPERIMENTAL REALIZATION OF THE OPTIMAL TRANSPOSE MAP}

In Section II the link existing between all optimal antiunitary operations has been considered. In the present Section a simple and significant implementation of the optimal approximation to the transpose map $K$ is reported by a different approach. The optimal transpose map $\mathcal{E}_{T R}$ has the following Kraus representation [24]

$$
\mathcal{E}_{T R}(\rho)=\frac{1}{3}\left(\mathbb{I} \rho \mathbb{I}+\sigma_{X} \rho \sigma_{X}+\sigma_{Z} \rho \sigma_{Z}\right)
$$

The action of the map $\mathcal{E}_{T R}$ can be viewed as the equiprobable occurrence of three different operators, $\mathbb{I}, \sigma_{X}$ and $\sigma_{Z}$. Such transformation can be achieved either by the action of an unitary operator into a larger system or by a stochastic evolution of the system.

Let us outline the importance of the $K$ and $\mathcal{E}_{T R}(\rho)$ transformations in the context of quantum information. The transpose map $K$ is a $P-$ map, as said. As such it transforms entangled states into non-physical ones. It is exactly this property that makes the transposition operation so important in all criteria of inseparability for two qubit systems. A bipartite state $\rho_{A B}$ of two qubits, $A$ and $B$, is entangled if and only if the density matrix $\mathbb{I}_{A} \otimes K_{B}\left(\rho_{A B}\right)$ has negative eigenvalues [35], where the operation $\mathbb{I}_{A} \otimes K_{B}$ is commonly referred to Partial Transpose operation. An experimental limitation of this criteria is that it requires complete knowledge of the state $\rho_{A B}$. Recently Horodecki and Ekert have found an experimental method for a direct detection of quantum entanglement exploiting the former criteria [36]. It consists of applying the map $\widetilde{\mathbb{I}_{A} \otimes K_{B}}=\left[\frac{1}{3} \mathcal{E}_{U N O T-A} \otimes \mathcal{E}_{D E P-B}+\frac{2}{3} \mathbb{I}_{A} \otimes \mathcal{E}_{T R-B}\right]$ to the state $\rho_{A B}$ where $\mathcal{E}_{D E P}(\rho)=$ $\frac{1}{4}\left(\mathbb{I} \rho \mathbb{I}+\sigma_{X} \rho \sigma_{X}+\sigma_{Y} \rho \sigma_{Y}+\sigma_{Z} \rho \sigma_{Z}\right)$ represents a depolarizing channel. Note, in the expression above, the appearance of both the optimal U-NOT map and of the 
optimal transpose maps. The measurement of the lower eigenvalue $\Lambda_{\min }$ of $\widetilde{\mathbb{I}_{A} \otimes K_{B}}\left(\rho_{A B}\right)$ is a syndrome of the separability of the state. In particular, $\Lambda_{\min } \leq \frac{2}{9}$ is found to imply entanglement [36]. In this framework it is important to achieve a high fidelity and reliable implementation of the stochastic optimal transpose map.

Let us consider the most general single qubit map based on the $4-\operatorname{dim}$ vectorial representation of the qubit density operator $\rho=\frac{1}{2}(\mathbb{I}+\vec{r} \cdot \vec{\sigma})$ written in terms of the vector $(1, \vec{r})$ in the 4 -dimensional space $\left\{\sigma_{0} \equiv \mathbb{I}, \sigma_{i}, i=1,2,3\right\}$. Any map $\mathcal{E}$ is fully characterized by a $4 \times 4$ real matrix $\mathbf{M}$, which maps $\rho$ into the density matrix: $\rho^{\prime}=\mathbf{M} \rho$. In particular any complete positive map has the following matrix representation

$$
\mathbf{M}=\left(\begin{array}{cc}
1 & 0 \\
\vec{t} & \mathbf{T}
\end{array}\right)
$$

where $\mathbf{T}$ is a $3 \times 3$ matrix and $\vec{t}$ is a $3-$ dim vector [37]. The $4 \times 4$ matrix $\mathbf{M}_{T R}$ associated with the map $\mathcal{E}_{T R}$ transpose reads

$$
\mathbf{M}_{T R}=\left(\begin{array}{cccc}
1 & 0 & 0 & 0 \\
0 & 1 / 3 & 0 & 0 \\
0 & 0 & -1 / 3 & 0 \\
0 & 0 & 0 & 1 / 3
\end{array}\right)
$$

We have implemented the optimal transpose gate by stochastically applying the identity $\mathbb{I}$ and Pauli operators $\sigma_{X}$ and $\sigma_{Z}$. In particular the random feature of the map was realized in an ergodic fashion in the time domain, by alternate on/off switching of suitable optical devices, indeed wp's, as shown shortly.

In the experiment carried out, the input qubit was codified into the $\pi$-state of a single photon and the Pauli operators $\sigma_{Z}$ and $\sigma_{X}$ were realized adopting $\lambda / 2$ waveplates respectively with angle setting $\theta$ equal to $0^{\circ}$ and $45^{\circ}$. A very general reconstruction of the optimal transpose transformation was experimentally attained adopting the well known Entanglement Assisted Quantum Process Tomography (EAQPT) [38]. This technique exploits the quantum parallelism associated with any entanglement process: the unknown map to be characterized acts on a subsystem of a bipartite entangled state and all the information about the map is obtained from the reconstruction of the transformed bipartite state. Indeed there is a one-to-one correspondence between the map and the final state. In this way only one input bipartite entangled state is needed to realize EAQPT.

Two entangled photons over the modes $k_{A}$ and $k_{B}$ in a singlet-state of polarization with common wl $\lambda=2 \lambda_{p}=$ $795 \mathrm{~nm}$ were created by SPDC in a $1.5 \mathrm{~mm}$ thick BBO NL crystal pumped by a mode-locked beam with wl $\lambda_{p}$ : (Fig. 7-a). We have fully characterized the implemented stochastic map $\mathcal{E}_{E X P}$ by reconstructing the associated representation matrix $\mathbf{M}_{E X P}$ by using of the input entangled state $\rho_{A B}$ of the two photons. The first step consisted of performing the quantum state tomography of the input system $\rho_{A B}$. In a following step the qubit associated with mode $k_{A}$, call it "qubit $A$ ", was left unchanged while the "qubit $B$ ", associated with the mode $k_{B}$ underwent the $\mathcal{E}_{E X P}(\rho)$-transformation. Note that this procedure implies the investigation of the unknown map $\mathcal{E}_{E X P}(\rho)$, by a complete span over the Hilbert space $\mathcal{H}_{B}$ of the injected "qubit $B$ " because of its fully mixed-state condition. The final state of the two qubits $\rho_{A B}^{\prime}=\mathbb{I}_{A} \otimes \mathcal{E}_{E X P-B}\left(\rho_{A B}\right)$ was again investigated by tomographic characterization. Finally, the matrix $\mathbf{M}_{E X P}$ was estimated by means of the experimentally determined density matrices $\rho_{A B}$ and $\rho_{A B}^{\prime}$ by adopting the relation: $\mathbf{M}_{E X P}^{T}=\mathbf{C}^{-1} \mathbf{C}^{\prime}$. In this expression $\mathbf{C}_{i, j=0,3}=\operatorname{Tr}\left[\left(\sigma_{i A} \otimes \sigma_{j B}\right) \rho_{A B}\right]$ and $\mathbf{C}_{i, j=0,3}^{\prime}=$ $\operatorname{Tr}\left[\left(\sigma_{i A} \otimes \sigma_{j B}\right) \rho_{A B}^{\prime}\right.$ are the measured correlation matrices used to reconstruct $\rho_{A B}$ and $\rho_{A B}^{\prime}$ respectively. We note that for an entangled state the matrix $\mathbf{C}$ is always invertible. The EAQPT reconstruction of $\mathbf{M}_{E X P}$ is shown in Fig.7-b.

In order to compare the matrix $M_{E X P}$ associated to the map $\mathcal{E}_{E X P}$ with the matrix $M_{T R}$ corresponding to the optimal transpose operation $\mathcal{E}_{T R}(23)$, we introduce the fidelity $\mathcal{F}(\mathcal{E}, \mathcal{L})=\int d \Psi F[\mathcal{E}(|\Psi\rangle\langle\Psi|), \mathcal{L}(|\Psi\rangle\langle\Psi|)]$ that quantifies the overlap between two generic maps $\mathcal{E}$ and $\mathcal{L} \quad[39,40]$. In the present context we obtain: $\mathcal{F}\left(\mathcal{E}_{T R}, \mathcal{E}_{E X P}\right)=1.01 \pm 0.01$. We may compare the diagram reported in Fig.7-b with the structure of the matrix $\mathbf{M}_{T R}$. The correspondence is quite impressive.

Note that the above stochastic transformation cannot be reversed, i.e. the initial state can not be restored and the information encoded in the output is lost in the environment. This is at variance with the optimal TeleUNOT Protocol described in Sections II and III [19] or the optimal UNOT gate based on the stimulated emission [12]. These transformations are indeed reversible: there the information about the input qubit, redistributed into several qubits (the flipped qubit and the two clones: ancilla qubits) can be reconstructed e.g. by a protocol suggested by Bruss et al. [41].

\section{CONCLUSIONS}

The Universal NOT gate and the Universal Optimal Quantum Cloning have been contextually implemented applying the projection over the symmetric subspace to the input qubit and to an appropriate ancilla system. This procedure has been found to consist of a modified quantum state teleportation scheme. All these protocols, extended to the case of an unlimited number of cloned or 
ancilla qubits, have been comprehensively and straightforwardly accounted by a novel, very general approach based on the well established angular momentum theory. By this approach many subtle connections with the programmable optimal teleportation of other more exotic anti-unitary transformations has been recovered. Most of these theoretical results have been substantiated by the corresponding experiments, also reported here. In particular, the linear (L) implementation of the teleportation of a quantum gate has been reported. It is an important tool to be adopted for the realization of complex QI networks since it allows to relax experimental constraints in order to achieve fault-tolerant processing [42]. Indeed the L-Optics quantum computation exploits the gate teleportation in order to transform a probabilistic computation into a nearly deterministic one [43]. Finally the stochastic feature of the optimal partial transpose has been experimentally characterized in the paper.

At last, it would be enlightening to compare the above results reported in this paper with the ones obtained recently by the adoption of the quantum injected nonlinear (NL) parametric amplifier (QIOPA) [17,12-14]. There the symmetrization procedure implied by cloning is provided automatically by the QED stimulated photon amplification process involving at the same time the injected qubit and the vacuum field. In other words, the QIOPA realizes symmetrization, cloning and entanglement within a unique fundamental, state-symmetrizing QED process. Furthermore, there the vacuum amplification may be thought to somewhat replace the mixed field associated with the mode $k_{A}$ in the linear $(\mathrm{L})$ symmetrization scheme shown in Fig. 4. In addition, the vacuum amplification provides the squeezed vacuum noise (SVN) that necessary affects in the QIOPA the deterministic realization of the non $\mathrm{CP}$ (cloning and UNOT) maps. In the $\mathrm{L}$ case, the non-probabilistic optimal realization of these maps replaces exactly the amount of lost information implied by SVN. In summary, the two conceptual approaches to cloning already discussed in Section I, i.e. the symmetrization and the QED amplification, appear to be connected by subtle quantum mechanical links. We believe to have enligthened in the present paper at least some of the most interesting of these links. We believe that the actual results, the suggestions and the open problems contributed by the present work could be useful at least by setting measurement bounds and fundamental performance limitations in the domain of Quantum Information and Quantum Estimation. This work has been supported by the FET European Network on Quantum Information and Communication (Contract IST-2000-29681: ATESIT), by Istituto Nazionale per la Fisica della Materia (PRA "CLON") and by Ministero dell'Istruzione, dell'Università e della Ricerca (COFIN 2002). F.S. acknowledges "Progetto Giovani Ricercatori"
(M.I.U.R.) for financial contribution.

[1] W.K. Wootters, and W.H. Zurek, Nature (London) 299, 802 (1982); D. Dieks, Phys. Lett. A 92, 271 (1982).

[2] K. Kraus, States, Effects and Operations (SpringerVerlag, Berlin 1983); J. Preskill, Lecture Notes on Quantum Computation, http://www.theory.caltech. edu/people/ph229/\#lecture. A completely-positive map (CP-map) $\Lambda(\rho)$ preserves positivity for: (a) any local state in the Hilbert space $H$, (b) when tensor-multiplied with the identical map $\mathbf{I}$ acting on any Hilbert space $K$, the extended map $\Lambda(\rho) \otimes \mathbf{I}$ is positive for any state in the entangled space $H \otimes K$, for any extension of $K$. A positive map ( $P$-map) only satisfies property (a).

[3] H. Bechmann-Pasquinucci, and N. Gisin, Phys. Rev. A 59, 4238 (1999).

[4] V. Bužek, M. Hillery, and R.F. Werner, Phys. Rev. A 60, R2626 (1999); N. Gisin, and S. Popescu, Phys. Rev. Lett. 83, 432 (1999).

[5] S. Massar, and S. Popescu, Phys. Rev. Lett. 74, 1259 (1995).

[6] N. Gisin, and S. Massar, Phys. Rev. Lett. 79, 2153 (1997).

[7] D. Bruss, A. Ekert, and C. Macchiavello, Phys. Rev. Lett. 81, 2598 (1998).

[8] V. Bužek, and M. Hillery, Phys. Rev. Lett. 81, 5003 (1998)

[9] V. Bužek, and M. Hillery, Phys. Rev. A 54, 1844 (1996); R. Derka, V. Buzek, and A. Ekert, Phys. Rev. Lett. 80, 1571 (1998).

[10] V. Bužek, S.L. Braunstein, M. Hillery, and B. Bru $\beta$, Phys. Rev. A 56, 3446 (1997).

[11] R.F. Werner, Phys. Rev. A 58, 1827 (1998).

[12] F. De Martini, V. Bužek, F. Sciarrino, and C. Sias, Nature (London) 419, 815 (2002).

[13] A. Lamas-Linares, C. Simon, J.C. Howell, and D. Bouwmeester, Science 296, 712 (2002).

[14] D. Pelliccia, V. Schettini, F. Sciarrino, C. Sias, and F. De Martini, Phys. Rev. A 68, 042306 (2003); F. De Martini, D. Pelliccia, and F. Sciarrino, Phys. Rev. Lett. 92, 067901(2004).

[15] S. Fasel, N. Gisin, G. Ribordy, V. Scarani, and H. Zbinden, Phys. Rev. Lett. 89, 107901 (2002).

[16] H.K. Cummins, C. Jones, A. Furze, N.F. Soffe, M. Mosca, J.M. Peach, and J.J. Jones, Phys. Rev. Lett. 88, 187901 (2002).

[17] F. De Martini, Phys. Rev. Lett. 81, 2842 (1998).

[18] C. Simon, G. Weihs, and A. Zeilinger, Phys. Rev. Lett. 84, 2993 (2000); F. De Martini, V. Mussi, and F. Bovino, Opt. Commun. 179, 581 (2000).

[19] M. Ricci, F. Sciarrino, C. Sias, and F. De Martini, Phys. Rev. Lett. 92, 047901 (2004). 
[20] J.I. Cirac, A.K. Ekert, and C. Macchiavello, Phys. Rev. Lett. 82, 4344 (1999).

[21] M. Ricci, F. De Martini, N. Cerf, R. Filip, J. Fiurasek, and C. Macchiavello, quant-ph/0403118.

[22] F. Sciarrino, C. Sias, M. Ricci, and F. De Martini, Phys. Lett. A 323, 34 (2004).

[23] C. Bennett, G. Brassard, C. Crepeau, R. Jozsa, A. Peres, and W. Wootters, Phys. Rev. Lett. 70, 1895 (1993).

[24] M.A. Nielsen and I.L. Chuang, Quantum Computation and Quantum Information (Cambridge, 2000); J. Preskill, Lecture Notes on Quantum Computation, http://www.theory.caltech. edu/people/ph229/ \#lecture.

[25] G. Brassard, PhysComp96 (eds Toffoli, Biafore and Leao) 48-50 (New England Complex Systems Inst., Cambridge, Massachusetts, 1996).

[26] M.A. Nielsen, E. Knill, and R. Laflamme, Nature (London) 396, 52 (1998).

[27] J.J. Sakurai, Modern quantum mechanics (AddisonWesley, 1985).

[28] F. Buscemi, G. M. D'Ariano, P. Perinotti, and M. F. Sacchi, Phys. Lett. A 314, 374 (2003).

[29] M. Hillery, V. Buzek, and M. Ziman, Phys.Rev. A. 65, 022301 (2002).

[30] A.R. Edmonds, Angular momentum in quantum mechanics (2. ed.) (N.Y.: Princeton University press, 1960).

[31] J. Casamiglia and N. Lutkenhaus, Appl. Phys. B: Lasers Opt. 72, 67 (2001).

[32] D. Boschi, S. Branca, F. De Martini, L. Hardy and S. Popescu, Phys. Rev. Lett. 80, 1121 (1998).

[33] W.T.M. Irvine, A. Lamas Linares, M.J.A. de Dood, and D. Bouwmeester, Phys. Rev. Lett. 92, 047902 (2004).

[34] C. Santori, D. Fattal, J. Vuckovic, G.S. Solomon, and Y. Yamamoto, Nature (London) 419, 594 (2002).

[35] M. Horodecki, P. Horodecki, and R. Horodecki, Phys. Lett. A 223, 1 (1996); A. Peres, Phys. Rev. Lett. 77, 1413 (1996).

[36] P. Horodecki, and A. Ekert, Phys. Rev. Lett. 89, 127902 (2002); P. Horodecki, Phys. Rev. Lett. 90, 167901 (2003).

[37] D.K.L. Oi, quant-ph/0106035.

[38] F. De Martini, A. Mazzei, M. Ricci, G.M. D'Ariano, Phys. Rev. A 67, 062307 (2003); G.M. D'Ariano and P. Lo Presti, Phys. Rev. Lett. 86, 4195 (2001).

[39] M.D. Bowdrey et al., Phys. Lett. A 294, 258 (2002).

[40] G.M. D'Ariano, and P. Lo Presti, Phys. Rev. Lett. 91, 047902 (2003); J.B. Altepeter, et al., Phys. Rev. Lett. 90, 193601 (2003).

[41] D. Bruss, J. Calsamiglia, and N. Lutkenhaus, Phys. Rev. A 63, 042308 (2001).

[42] D. Gottesman, and I.L. Chuang, Nature (London) 402, 390 (1999).

[43] E. Knill, R. Laflamme, and G. Milburn, Nature (London) 409, 46 (2001).

\section{Figure Captions}

Figure.1 General scheme for the simultaneous realization of the Teleportation of the UNOT gate (Tele-UNOT) and the Universal Quantum Cloning Machine (UOQCM) by applying a projective operator. The optional insertion on the channel $A$ of a suitable unitary operator $U$ allows the optimal teleportation of any anti-unitary map at Bob's site.

Figure.2. Realization of the UOQCM and of the TeleUNOT gate by means of a quantum circuit.

Figure.3. General scheme for the simultaneous realization of the $N \rightarrow(M-N)$ Tele-UNOT gate $($ Tele $-U N O T)$ and of the $N \rightarrow M$ UOQCM by applying the statesymmetrization projective operator.

Figure.4 Setup for the optical implementation of the $\mathrm{Ou}$ Mandel Cloning and corresponding results for three input qubits. Filled squares: plots corresponding to the "correct" polarization; Open triangles: plots corresponding to the "wrong" polarization. The solid line represents the best gaussian fit expressing the correct polarization. These options also apply to Figures 5 and 6 .

Figure.5. Setup for the optical implementation of the Cloning process in a modified teleportation scheme. The corresponding results for three input qubits are also reported.

Figure.6. Setup for the optical implementation of the Tele-UNOT gate and corresponding results for three input qubits.

Figure.7. Setup for the optical implementation of the Entanglement Assisted Quantum Process Tomography $(E A Q P T)$ of a stochastic map implementing the optimal partial transpose (a). Experimental reconstructed matrix operation $\mathbf{M}_{E X P}(\mathbf{b})$. 


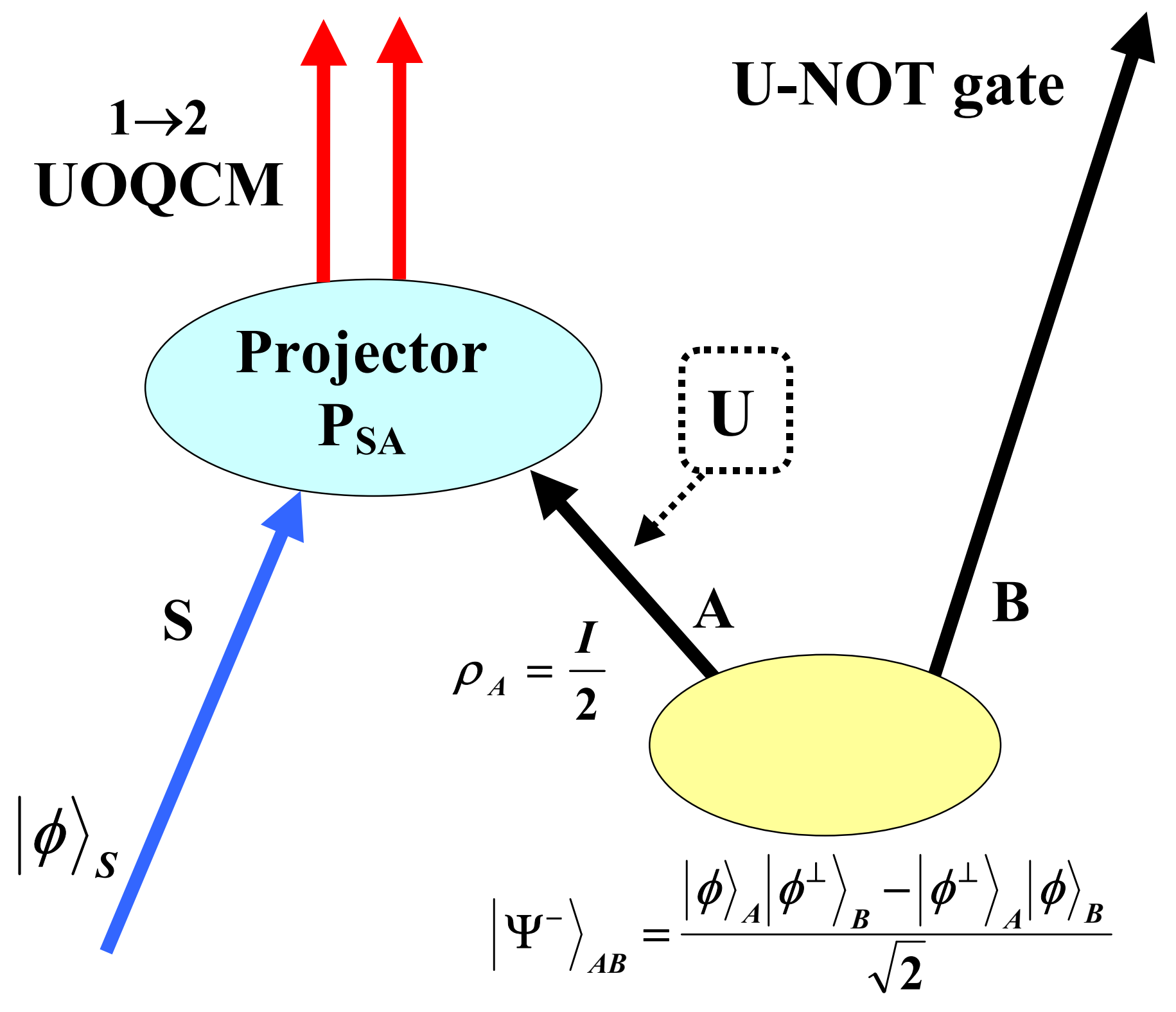




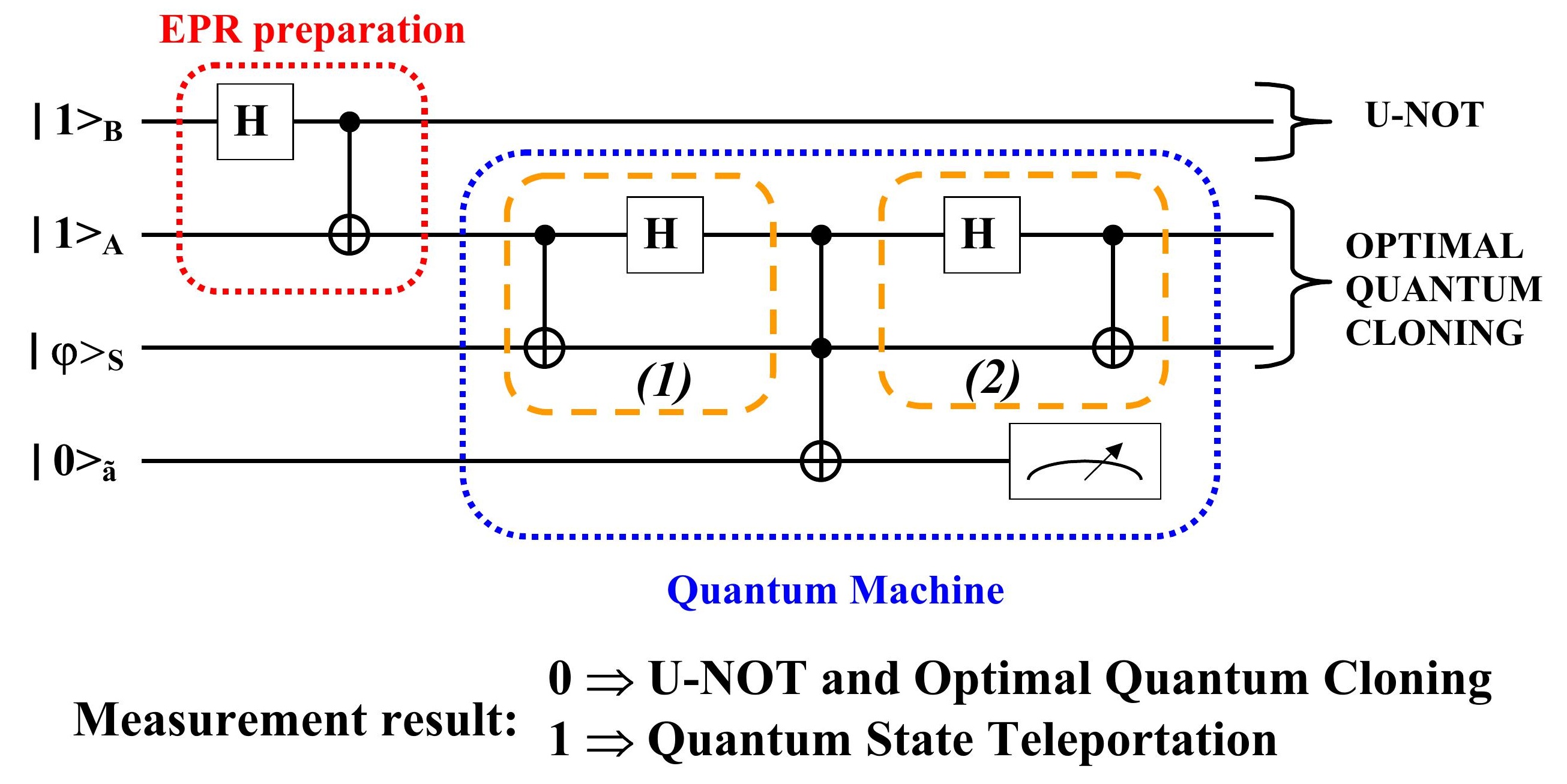




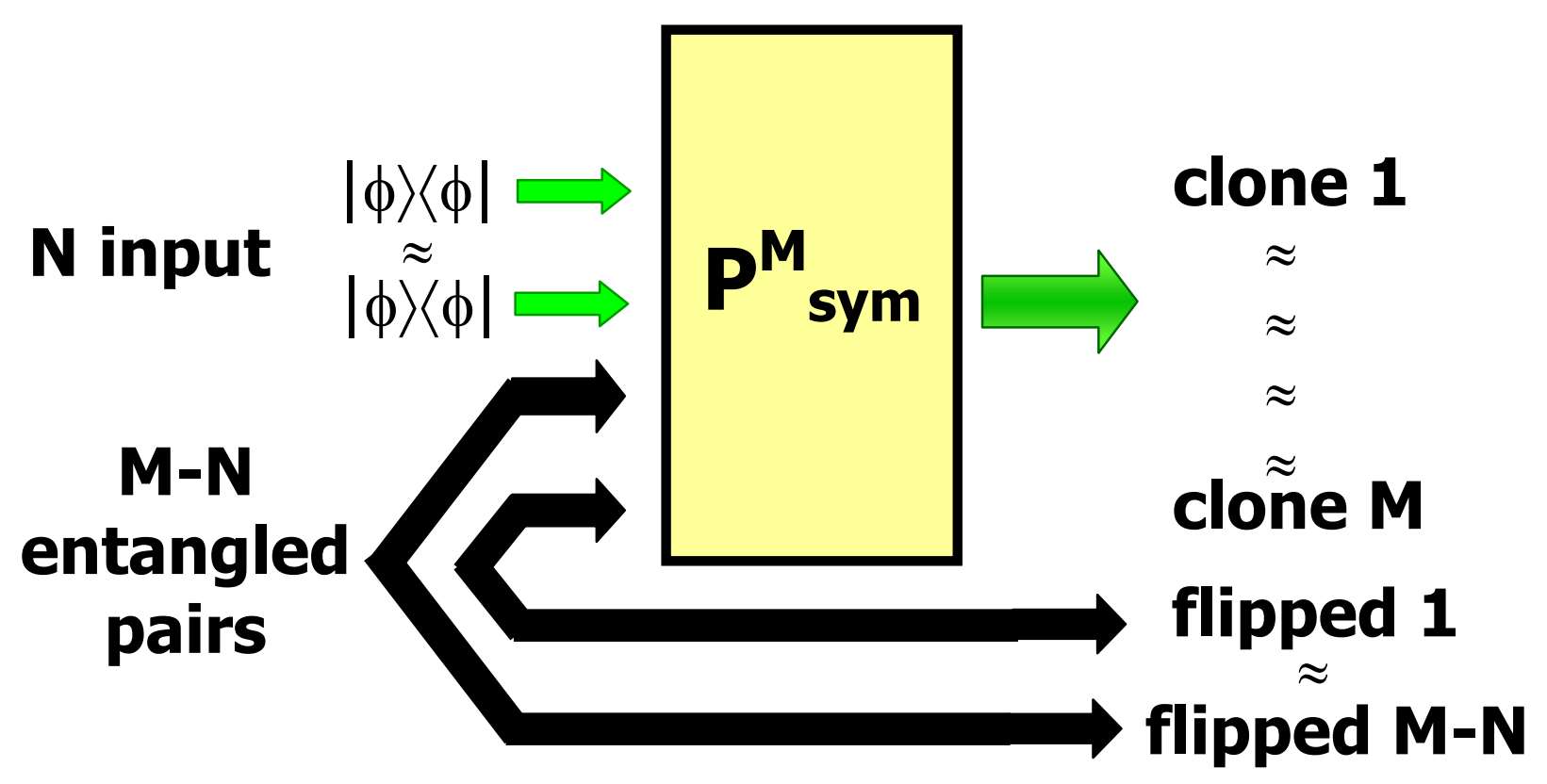




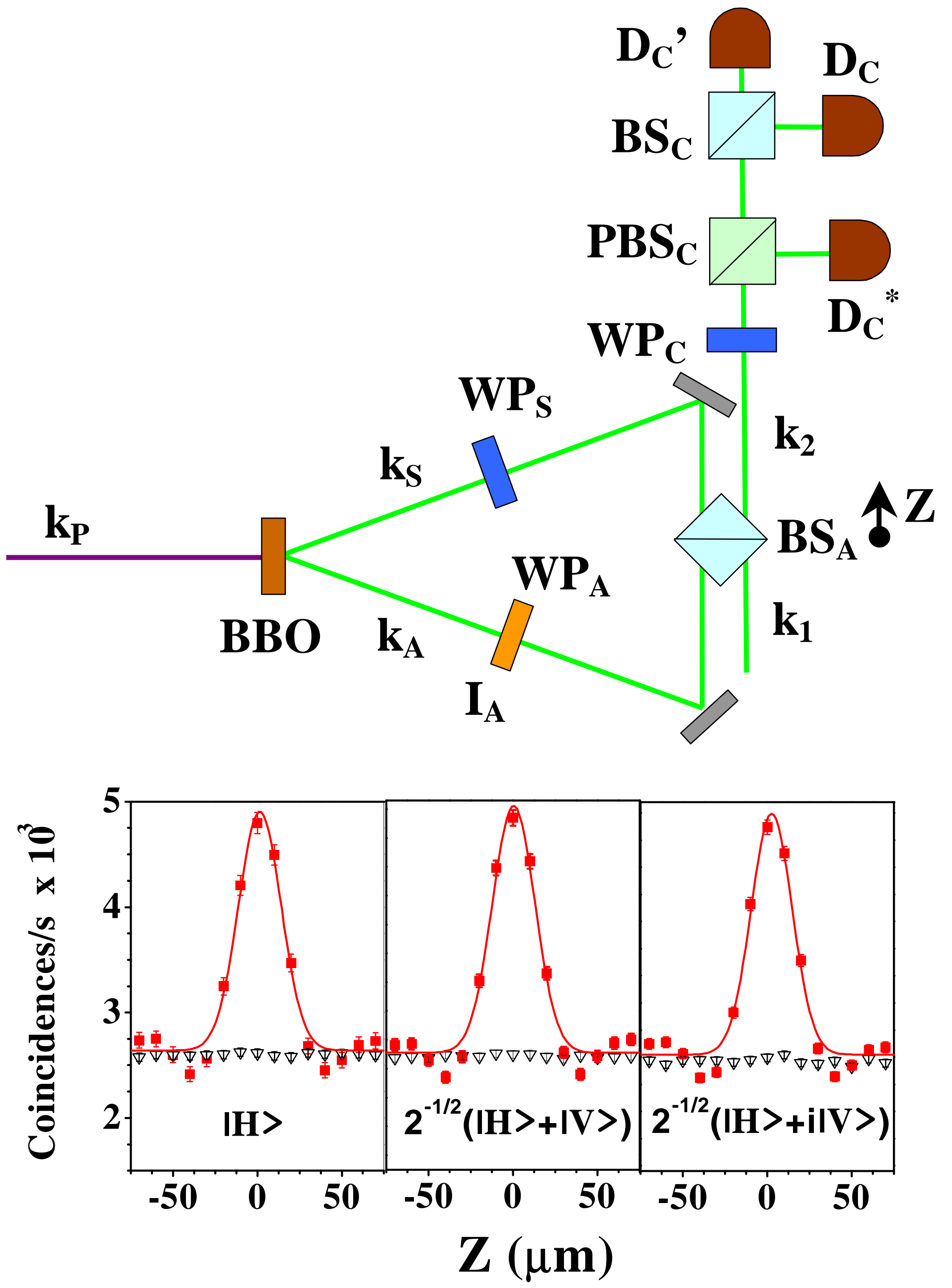



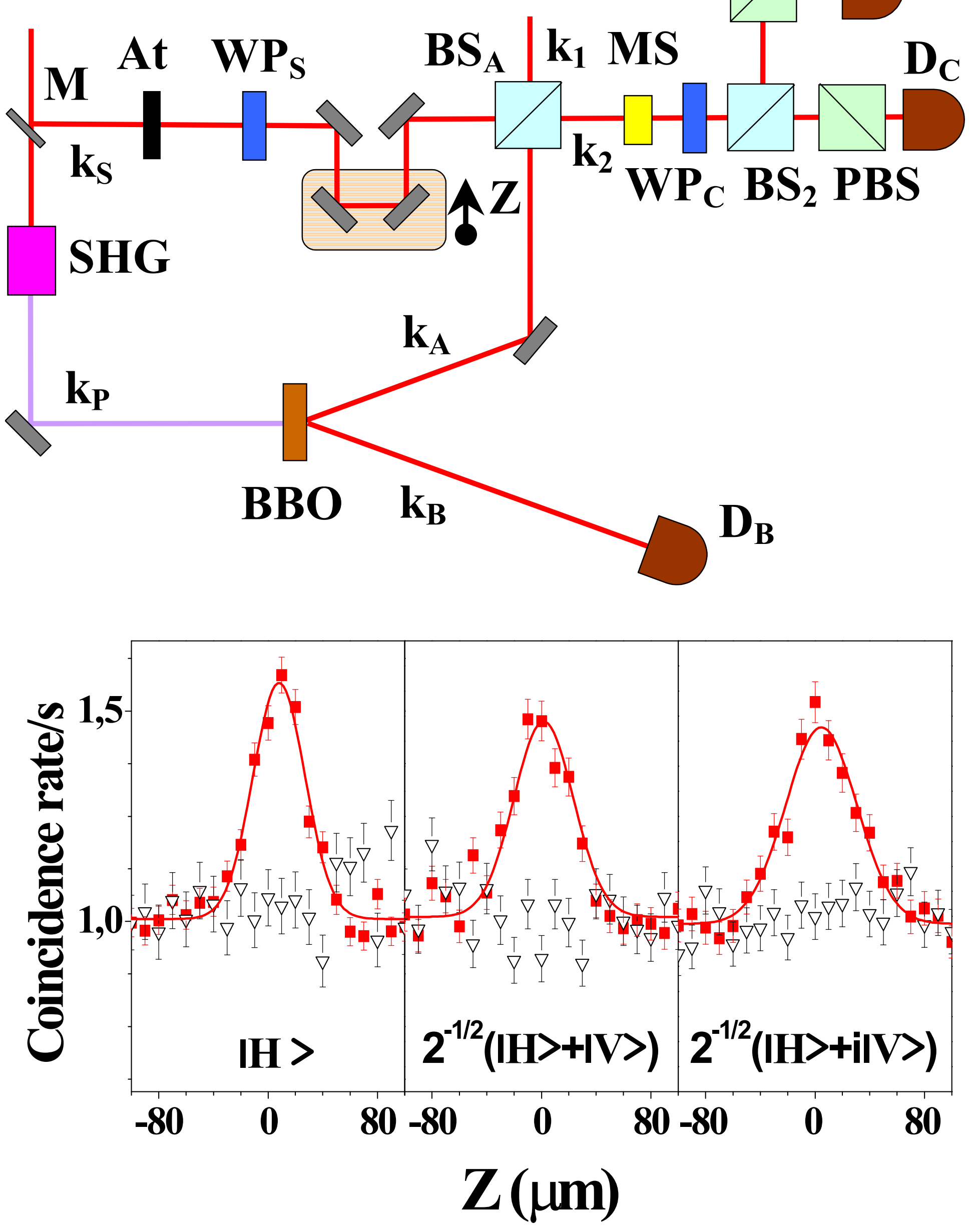

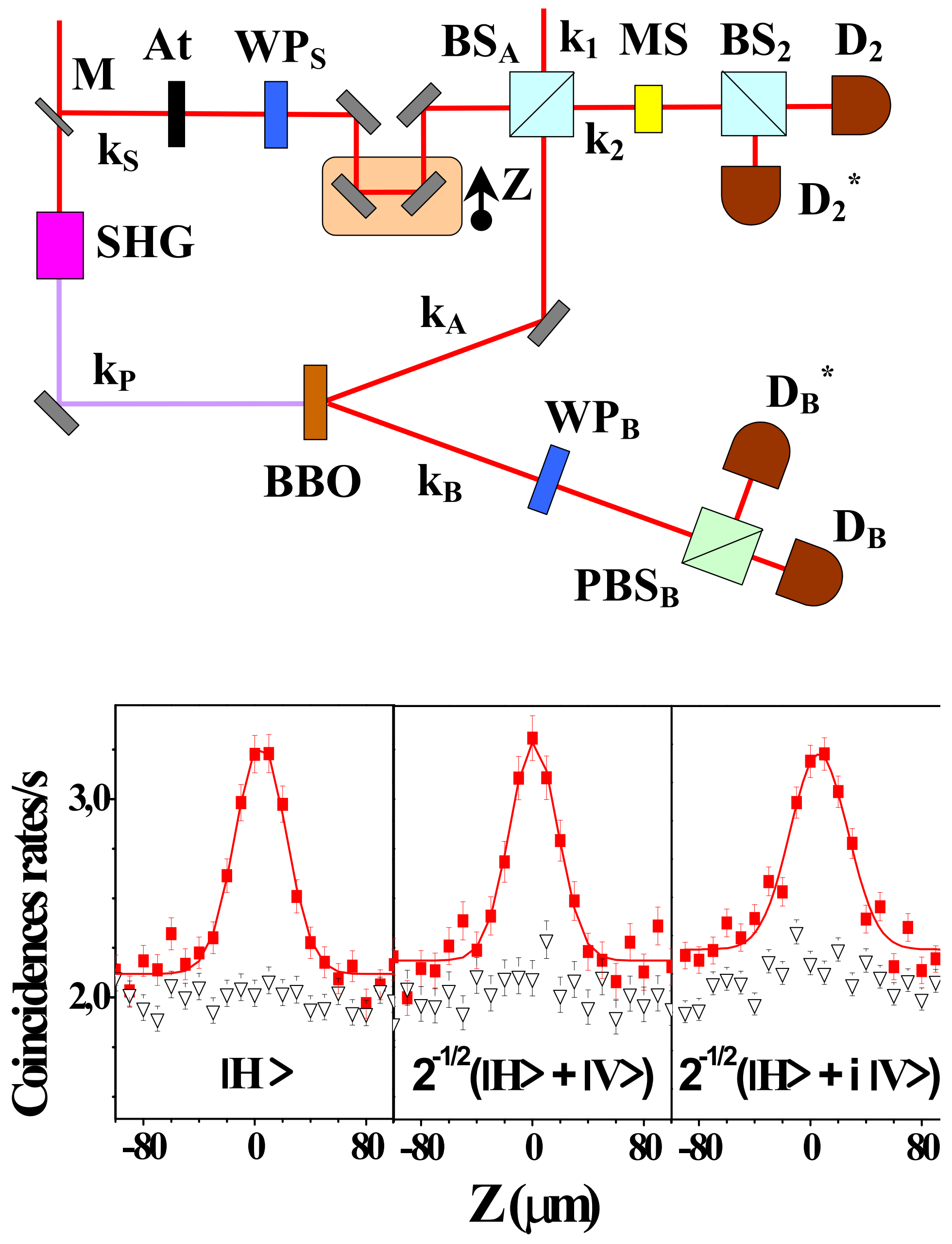
a)

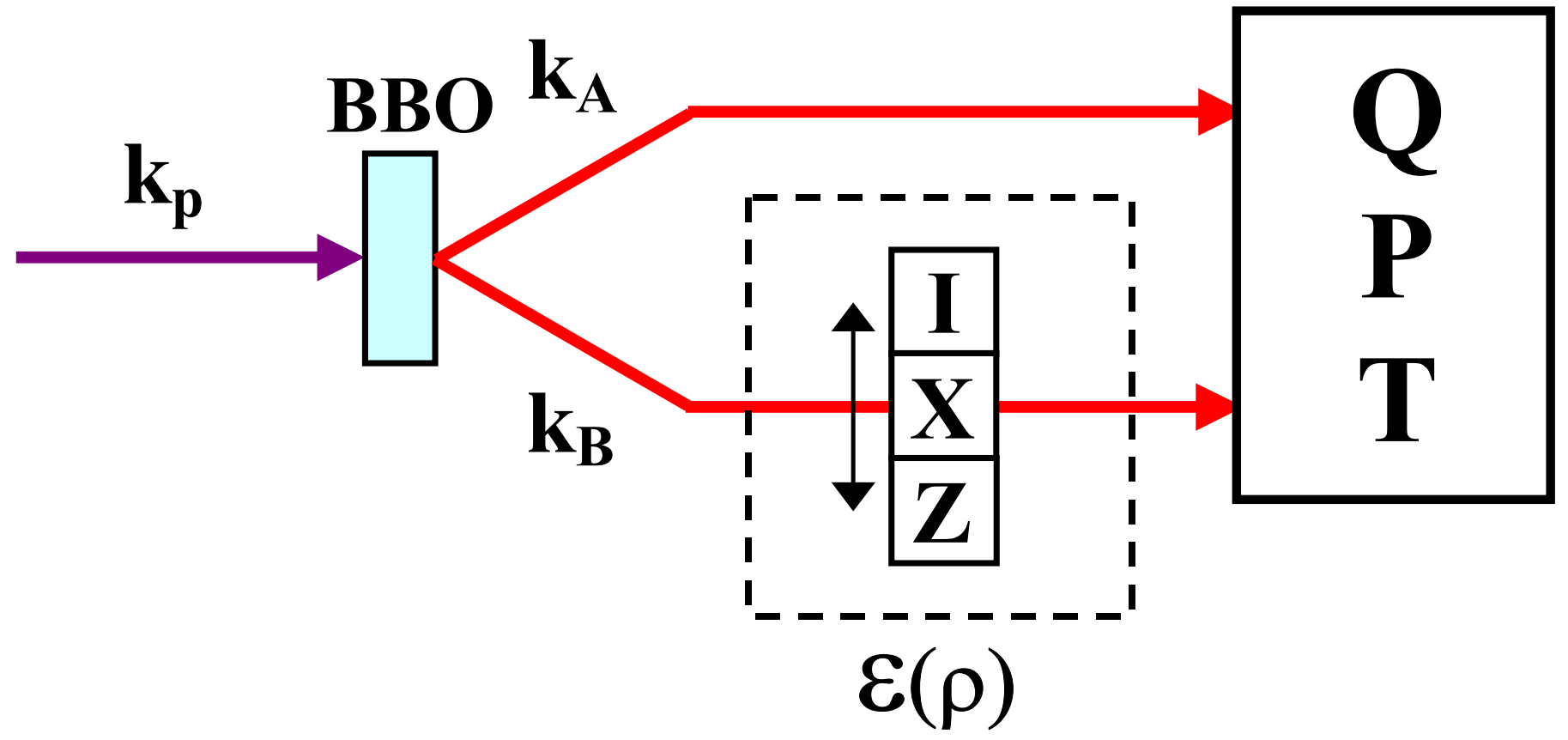

b)

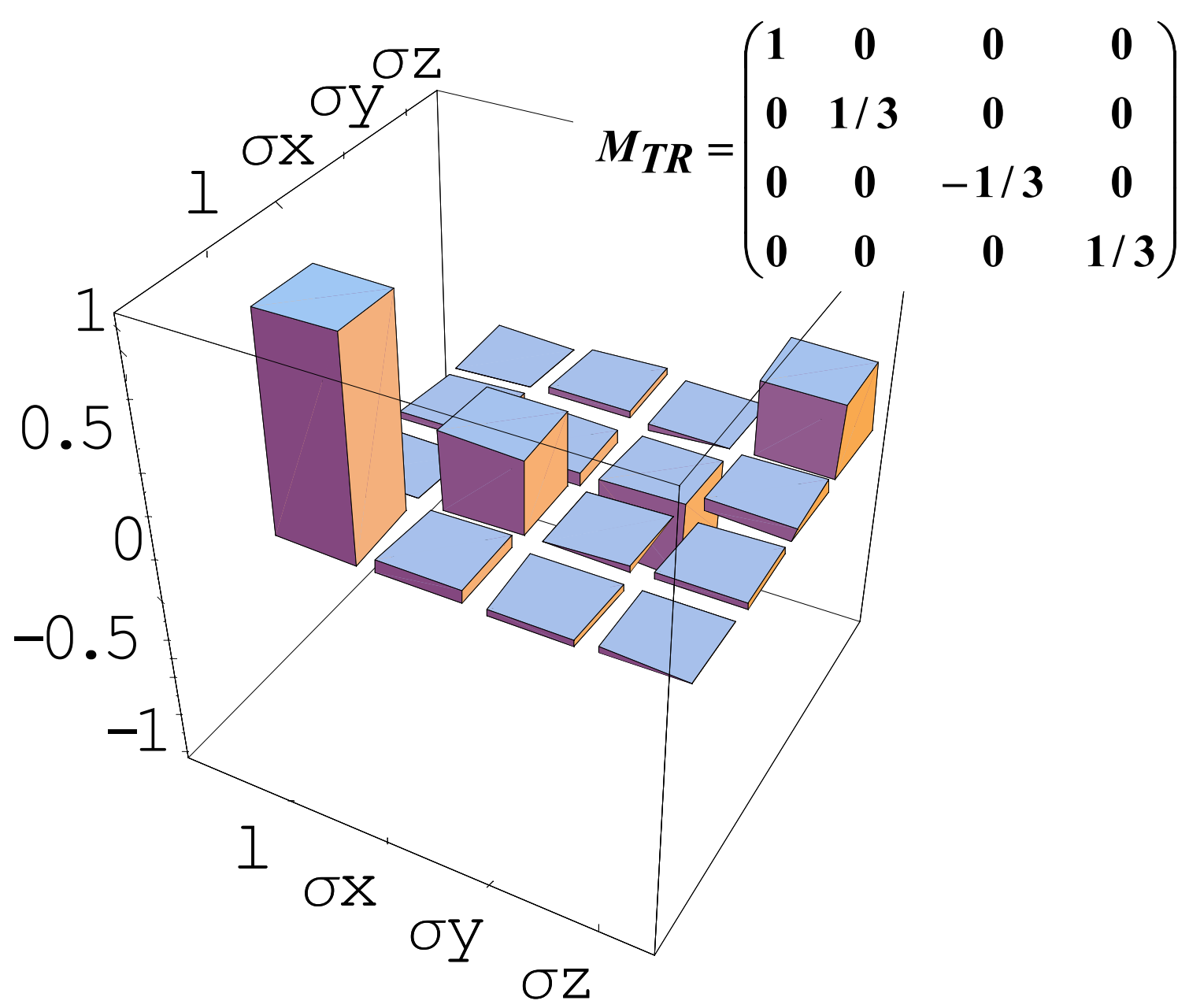

